\title{
AN EXPERIMENTAL AND NUMERICAL STUDY OF THE INFLUENCE OF LOCAL EFFECTS ON THE APPLICATION OF THE FIBRE PUSH-IN TESTS.
}

\author{
Jon M. Molina-Aldareguía ${ }^{1}$, M. Rodríguez ${ }^{1}$, C. González ${ }^{1,2}$ and J.LLorca ${ }^{1,2}$ \\ ${ }^{1}$ Madrid Institute for Advanced Studies of Materials (IMDEA-Materials) \\ C/ Profesor Aranguren s/n, 28040 - Madrid, Spain \\ ${ }^{2}$ Departamento de Ciencia de Materiales, E.T.S. de Ingenieros de Caminos, \\ Canales y Puertos, Universidad Politécnica de Madrid, C/ Profesor Aranguren s/n, \\ 28040 Madrid, España. \\ E-mail: m.rodriguez@imdea.org
}

\begin{abstract}
Several methods have been developed to test interfacial adhesion in composite materials such as pull-out, microbond and push-in/push-out tests. Some of them can only be applied to single fibre matrix composites and others are difficult to perform on brittle fibres due to premature fracture of the fibre. Push-in tests, consisting on pushing the fibre with a micro or nanoindenter on a bulk specimen, constitute a powerful technique that can be applied directly on composite laminates. However, the interfacial adhesion values obtained from different tests (microbond, push in) often differ and even are subjected to a large scatter. This might be due to the fact that the existing analytical solutions that are typically used to interpret the experimental data take into account the constrain effect of the surrounding fibres on a simplified manner. To study this, we have carried out a careful micromechanical modelling of the push-in test, coupled with experimental adhesion testing in a glass fibre reinforced epoxy matrix composite. The model takes into account the interfacial fracture process by means of interface cohesive elements at the fibre-matrix interface and focuses on the study of the constrain effects due to the local configuration of the surrounding fibres.
\end{abstract}

KEY WORDS: matrix composites; interfacial strength; push-in test; nanoindentation

\section{INTRODUCTION}

Advanced computational micromechanics has demonstrated its potential to predict the constitutive response of fibre-reinforced composites from the properties of matrix, fibre and their interface [1], [2]. To quantify the interfacial properties, several micromechanical methods exist to test interfacial adhesion in composite materials such as the pull-out test, the microbond test and the push-in and push-out tests. This kind of tests present an ideal interfacial adhesion measure since they carried out on real composite laminates and not on samples fabricated with a particular geometry for such a purpose. This will allow establish the degradation of the interface properties on the real composite under service conditions, as it is widely known that these composites are particularly sensitive to exposition to hot-wet conditions [3]. Other tests suffer from several experimental difficulties: the pull-out test is not easy to perform on brittle fibres due to premature fracture of the fibre and the push-out test [4], [5], requires the preparation of thin membranes $(\sim 50 \mu \mathrm{m})$ of the composite material prior to testing.

In this situation, the push-in test, consisting on pushing one fibre with a micro or nanoindenter on the crosssection of a bulk specimen, [6]-[9], constitutes a powerful technique that can be applied directly on real composite laminates, either before or after degradation under service conditions. Nevertheless, the evaluation of interfacial properties remains a controversial issue among researchers of composite materials due to experimental partly due to the fact that the existing analytical solutions typically used to interpret the experimental data are based on simplified shear lag analytical models [10], [11]. As such, these models only take into account the constrain effect of the surrounding fibres in a simplified way, but the neighboring fibres are distributed in irregular configurations and no solution exists for the stress distribution around an indented fibre in a non-axisymmetric configuration. The axisymmetric models used involve an averaged distance to the nearest-neighbour fibres, which are usually considered perfectly rigid [8], [9]. As such, the derived parameters to account for the effect of neighbour fibres remain somewhat arbitrary.

To overcome this limitation, we have carried out a careful micromechanical modelling of the push-in test, coupled with experimental adhesion tests in a glass fibre reinforced epoxy matrix composite using nanoindentation. The model addresses the interfacial fracture process through the use of interface cohesive elements at the fibre-matrix interface. The study focuses on the constrain effects due to the local configuration of the surrounding fibres and helps to determine the validity of the simplified shear lag models currently used to interpret the results. It is shown that the analytical models are valid when the indented fibre is relatively isolated from its neighbours, but that they tend to overestimate the constrain effect for closely 
packed fibres, the scenario usually found in polymer matrix composites. Nevertheless, it is concluded that the simplified shear lag models can be safely used to estimate the interfacial shear strength provided that the constrain effect is carefully derived from the initial part of the push-in loading-displacement curve.

\section{MATERIALS AND EXPERIMENTAL TECHNIQUES}

A unidirectional glass/epoxy composite was used for this study. Pre-impregnated sheets of E-glass/MTM 57 epoxy resin were purchased from Advanced Composite Group (UK). Rectangular panels of $350 \times 300 \mathrm{~mm} 2$ were heated at $3{ }^{\circ} \mathrm{C} / \mathrm{min}$ and consolidated at $120^{\circ} \mathrm{C}$ and $0.64 \mathrm{MPa}$ in an autoclave for 30 minutes. They were cooled at the same rate of $3{ }^{\circ} \mathrm{C} / \mathrm{min}$ and the internal pressure was released at $80{ }^{\circ} \mathrm{C}$. The nominal fibre volume fraction was $54 \%$. Blocks of 1 x 1 x $1 \mathrm{~cm}$ were cut and polished on $\mathrm{SiC}$ paper to 1000 grit finish followed by a diamond slurry up to $1 \mu \mathrm{m}$. The samples were mounted on a MTS Nanoindenter XP instrumented equipped with a Berkovich tip. Fibres covering a wide range of diameters (between 14 and $32 \mu \mathrm{m}$ ) and different environments (position of the neighbouring fibres) were selected for the tests, as shown in two examples in figure 1 .

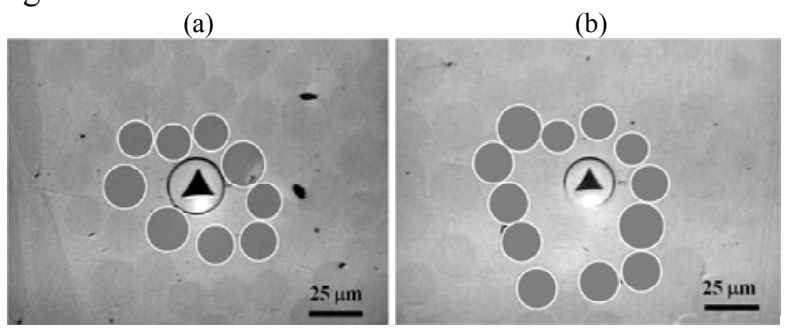

Figure 1. Examples of indented fibres showing the local configuration of the surrounding fibres: (a) a closely packed fibre and (b) a more isolated fibre.
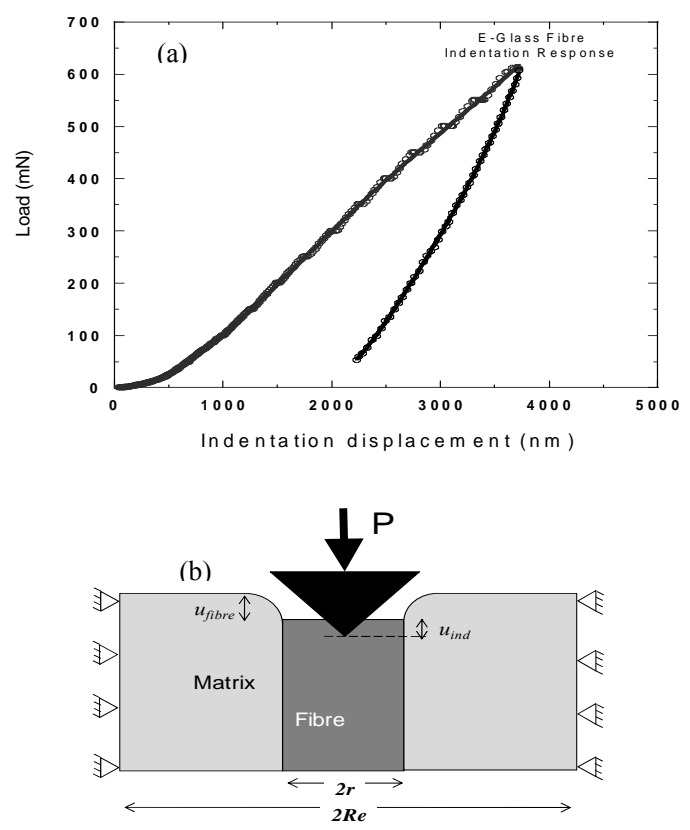

Figure 2. (a) Typical indentation load-displacement curve performed on a glass fibre and (b) sketch illustrating the indentation response of the fibre showing the two components of the indenter displacement: the penetration of the indenter in the fibre cross-section $u_{\text {ind }}$ and the compression of the fibre and deflection of the matrix $u_{\text {fibre }}$.

The push-in test consists of pushing the indenter into the cross-section of a fibre which is perpendicular to the polished section. The indentations were performed at a displacement rate of $50 \mathrm{~nm} / \mathrm{s}$ at various levels of maximum load ranging between $20 \mathrm{mN}$ and $700 \mathrm{mN}$.

Figure 2 (a) shows a typical load-displacement curve obtained during indentation of one fibre. As shown previously in polymer matrix composites [12] no significant discontinuity in the load-displacement curve is observed at the onset of debonding. Several authors [8], [9], have thoroughly work on the treatment of the indentation curves in order to obtain a debonding load without the need for time-consuming load step procedures and optical inspection of the indented fibres. But for the sake of clarity, the most important aspects are briefly discussed below in conjunction with the experimental results obtained in this work.

\section{EXPERIMENTAL RESULTS}

\subsection{Extraction of load-fibre displacement curve}

The treatment relies on the identification of two components in the indenter displacement. The first is related to the penetration of the indenter in the fibre crosssection $u_{\text {ind }}$, and the second is associated with the compression of the fibre in the surrounding epoxy matrix and the deflection of the matrix $u_{\text {fibre, }}$ (figure 2 (b). In order to derive the elastoplastic indentation response of E-glass one can either indent a piece of bulk glass or derive it from finite element simulations. The second approach was used in this work.

Once the elastoplastic indentation response of bulk glass is known, the fibre displacement $u_{\text {fibre }}$ can be simply obtained by subtracting the elastoplastic component of indentation $\left(u_{\text {ind }}\right)$ from the total displacement $u$ recorded during the test, as shown in figure 3 (a). The curve in figure 3 (b) shows the typical behaviour found elsewhere [8], [9], for the fibre compression once the displacement due to the elastoplastic indentation is removed.

The initial linear part corresponds to the elastic response of the fibre/matrix system, while most authors interpret the departure from linearity at the critical load $P_{\text {crit }}$ as the result of interfacial debonding. In this work, the test was interrupted at increasing loads for optical inspection of the indented fibres.

As shown in figure 3 (c), it was difficult to establish whether the departure from linearity marked the onset of plastic deformation of the matrix or the onset of interfacial debonding, especially because debonding was gradual and did not occur on the entire periphery of the fibre simultaneously. 

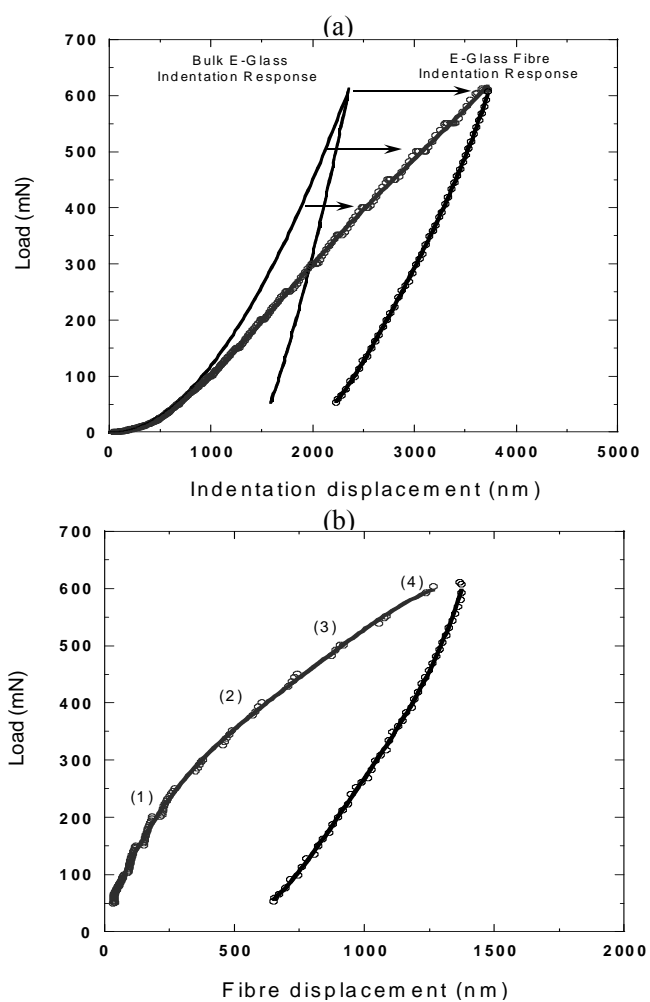

(c)

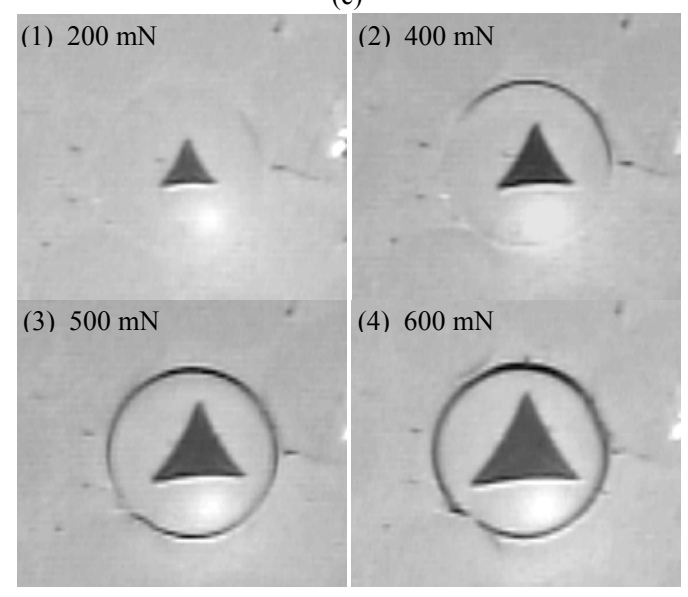

Figure 3. (a) Load-displacement curve of E-glass fibre and bulk glass (as predicted by FEM); (b) the load-fibre displacement behaviour obtained by subtracting the elastoplastic component of indentation $\left(u_{\text {ind }}\right)$ from the total displacement $u$ recorded during the test; (c) optical images of the indented fibre showing that the debonding is gradual.

\subsection{Analytical shear lag model}

The analytical shear-lag models currently used to interpret the load-displacement curves obtained in the push-in test assume a perfect hexagonal fibre packing, elastic deformation of the matrix and a perfectly bonded interface. The proximity of the surrounding fibres is taken into account through the parameter $R e$, as illustrated in figure 2(b), which represents the distance from the indented fibre to the ring of neighbouring fibres. The model assumes that the longitudinal displacement of the matrix equals zero at distance $R e$. Under this assumption, a linear relationship is obtained between the displacement of the fibre $u_{f}$ and the applied load $P[8]$, [9].

$$
u=\frac{P}{\pi \cdot r \cdot n \cdot E_{f}}(1) \quad \text { with } \quad n^{2}=\frac{2 G_{m}}{E_{f} \ln \left(\mathrm{R}_{\mathrm{e}} / r\right)}
$$

where $r$ is the fibre radius, $\sigma_{f}$ is the fibre axial stress, $G_{m}$ is the matrix shear modulus and $E_{f}$ is the fibre Young's modulus. Finally, the maximum shear stress at the onset of debonding can be assimilated to the interfacial shear strength:

$u=\frac{n \cdot P_{c r i t}}{2 \pi \cdot r^{2}}=\frac{n}{2} \sigma_{f}^{c r i t}$

where $\sigma_{f}^{\text {crit }}$ is the axial stress at the cross-section of the fibre at debonding. It is interesting to note that the analytical model tries to account for the effect of the surrounding fibres through the parameter $(\mathrm{Re} / \mathrm{r})$. This parameter $(R e / r)$ has no physical interpretation but it has been shown to correlate to the local packing of the indented fibre and can be denoted as the constraint factor. In the treatment proposed in [8], the linear part of the indentation curve is used to estimate $n$ and (Re/r) through equations (1) and (2). Once $\mathrm{n}$ is determined, the interfacial strength can be derived from the critical load for debonding $P_{\text {crit }}$ through equation (3).

\subsection{Effect of fibre diameter and neighbouring fibres}

The shear-lag model presented above was applied to the experimental results obtained in this work. Overall over 30 fibres were tested. Figure 4 (a) displays seven representative load-displacement curves, showing the large differences that can be found for fibres of different diameters or located in different environments (for the sake of clarity only the loading portions of the curves are displayed). As mentioned above, the linear part of the curves was used to estimate the constraint factor $(R e / r)$ through equations (1) and (2). As expected from equation (1), the slope of the linear part in this graph was constant for all the fibres, but the onset of departure from linearity was very sensitive to the local environment of the fibres.

Figure 4 where the load was normalized by the fibre diameter and $n$ clearly shows the behaviour of fibres with similar diameters. Fibres with a smaller constraint factor $R e / r$, i.e. having more closely packed fibres around, showed a more marked departure from linearity, corroborating the strong effect of the local environment of the neighbouring fibres on the push-in test.

Finally, the calculated interface shear strength (calculated from the load at the onset of the departure from linearity) is plotted in figure 5 as a function of the constraint factor. An average value of $83 \mathrm{MPa}$ was obtained although the uncertainty was large due to the difficulty on determining the critical load. The axial fibre stress at the onset of debonding varied linearly with the constraint factor (figure 5), as expected. 

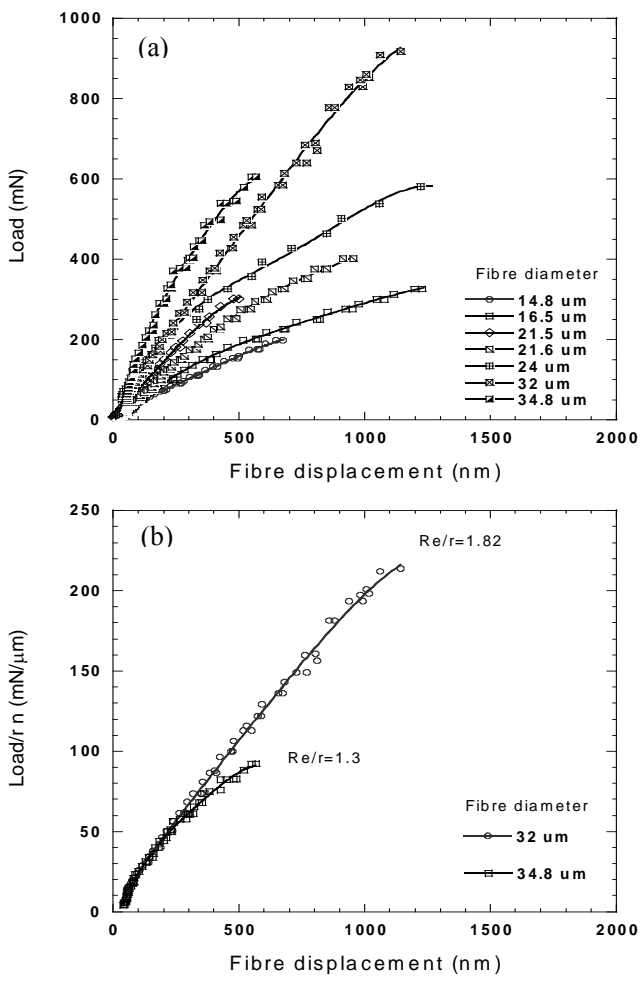

Figure 4. (a) Load-fibre displacement behaviour for some representative fibres of different diameter; (b) Normalized load curves for fibres with 32-35 $\mu \mathrm{m}$ diameter showing the effect of the confinement parameter Re/r.

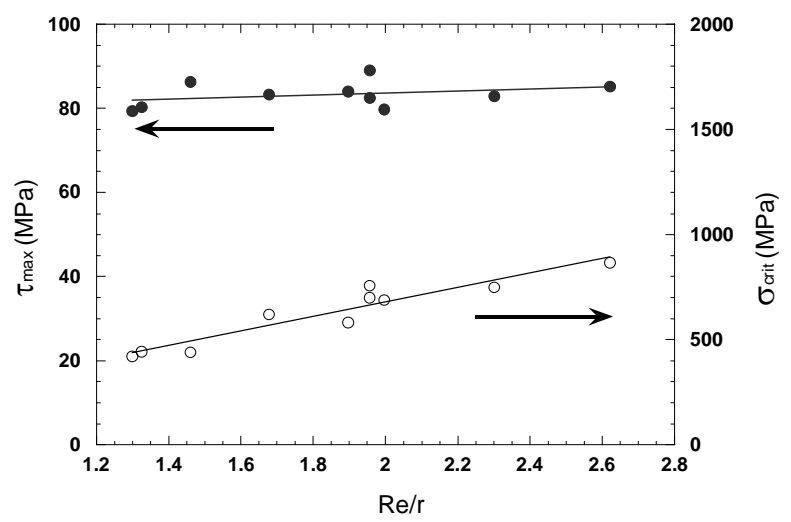

Figure 5. Interface shear stress and axial fibre stress at the onset of departure from linearity as a function of the constraint factor.

It is interesting to note that the ratio $\mathrm{Re} / \mathrm{r}$ can be related to the fibre volume fraction $V_{f}$ under the assumption of hexagonal fibre packing according to:

$\ln (R e / r)=\frac{1}{2} \ln \left(2 \pi / \sqrt{3} V_{f}\right)$

which for a nominal fiber volume fraction of $54 \%$ leads to $R e / r=2.6$. The fact that the reported values are considerably lower reflects that the configuration of the fibres was far from the ideal hexagonal packing, as can be clearly seen in figure 1 . In fact, the constraint factors obtained were very small and one should wonder whether this approach is valid for composites with very high volume fractions of fibres. In addition, it was difficult to establish whether the onset of departure is due to interface decohesion or local plastic deformation of the matrix, which has a shear yield stress of $80 \mathrm{MPa}$, similar to the interfacial strength. To elucidate this, the validity of the approach is discussed in the following sections based on the results of finite element simulations.

\section{MODELLING}

The experimental results above demonstrate that the effect of the local environment of the surrounding fibres is not negligible, especially in cases where the volume fraction of fibres is very large. Advanced numerical simulations that take into account the details of the microstructure are thus critical to understand the role played by the surrounding fibres on the test results and to determine the minimum distance from the tested fibre to its nearest neighbours to minimize the constraint effect on the measured interfacial properties. The modelling strategy to simulate the push-in test is depicted in Fig. 6.

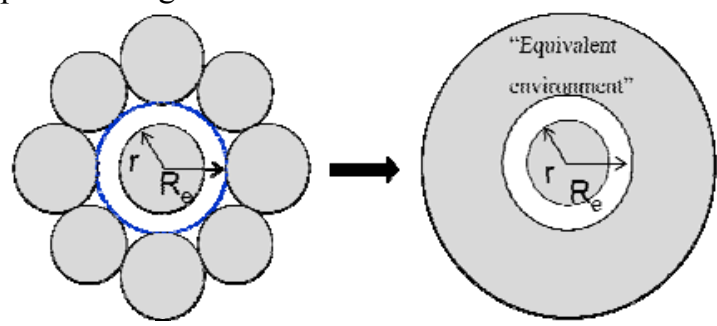

Figure 6. Simplification assumed in this work to model the effect of the local environment of the fibres as a solid ring with the same properties than the fiber located at a distance Re from the indented fibre.

For the sake of simplicity, the effect of the surrounding fibres in (a) at a distance $R e$ from the centre of the fibre has been modelled as a solid ring, displaying the same properties that the fibres. A fibre diameter of $20 \mu \mathrm{m}$ was assumed and the position of the solid ring was varied in the range $\operatorname{Re} / r \in(1.0, \infty)$ to account for the constraint effect of the neighbouring fibres.

The numerical analysis of the indentation of the fibre cross-section was carried out using the finite element method. The simplification depicted in figure 6 allowed for the implementation of an axisymmetric model. The fibres and matrix were meshed using two-dimensional four-node linear axisymmetric elements with reduced integration. The Berkovich indenter was modelled as a rigid conical indenter with an included angle of $70.3^{\circ}$. The specimen dimensions were large enough so that the effects of the boundary conditions were negligible and the mesh was refined near the indented zone, to avoid numerical instabilities at the contact between fibre and indenter. Glass fibres were modelled as linear elastoplastic isotropic solids using a Von Mises plasitification criterion, with the properties given in Table 1. The yield stress of the glass fibres was fixed by fitting the numerical simulations to the indentation behaviour of the fibres. The MTM57 epoxy matrix was 
assumed to behave as an isotropic, elastoplastic solid. The matrix elastic constants were obtained from the data sheet of ACG and are reported in Table 1. The yield stress of the MTM57 epoxy matrix was fixed at 140 $\mathrm{MPa}$ by fitting numerical simulations to the indentation behaviour of the matrix [13], giving a shear yield stress of $80 \mathrm{MPa}$.

\section{Table 1. Material Properties}

\begin{tabular}{|c|c|c|c|}
\hline Material & $\begin{array}{c}\mathrm{E} \\
(\mathrm{GPa})\end{array}$ & $v$ & $\begin{array}{c}\sigma_{\mathrm{y}} \\
(\mathrm{MPa})\end{array}$ \\
\hline E-Glass & 70 & 0.18 & 4200 \\
\hline $\begin{array}{c}\text { MTM57 } \\
\text { epoxy }\end{array}$ & 3.4 & 0.35 & 140 \\
\hline
\end{tabular}

Interface decohesion was simulated through a cohesive crack model, using first-order interface elements of Abaqus (COHAX4) inserted at the fibre/matrix interface which follow a traction-separation $(t-\delta)$ constitutive law. In the absence of damage, the interface behaviour was linear with an initial stiffness large enough to ensure the displacement continuity at the interface and to avoid any modification of the stress fields around the fibres in the absence of damage. This linear behaviour ends at the onset of damage, which occurs when the traction acting on the interface reaches the interface strength, $t_{c}$.

From the viewpoint of the interface properties, the interface behaviour is controlled by two parameters, namely the interface strength tc and the fracture energy, $G_{i}$, which stands for the area under the curve $\mathrm{t}-\delta$. The interface strength was fixed at $t_{c}=80 \mathrm{MPa}$, based on the preliminary experimental results obtained based on the shear-lag model. Regarding the interface fracture energy, this parameter should not significantly affect the onset of debonding and a value of $100 \mathrm{~J} / \mathrm{m} 2$ was used, as in previous analyses [14].

\section{NUMERICAL RESULTS AND COMPARISON WITH EXPERIMENTS.}

\subsection{Effect of the surrounding fibres on the linear slope of the loading curve}

The load-displacement curves obtained by FEM, for fibres with different constraint factors $R e / r$ are plotted in figure 7 assuming a perfect interface. As in the experiments, an initial linear response was obtained and the stiffness increased with the constraint of the surrounding fibres (lower $R e / r$ ). It is interesting to notice that the curves departed earlier from linearity as the constraint increased, marking the onset of plastic deformation of the matrix around the fibres (indicated by the gray arrows in the figure). At this point, the shear stresses reached $\tau_{\text {matrix }}=80 \mathrm{MPa}$, as expected from the matrix yield stress (table 1).

Comparison between the FEM results and the results that would be obtained on applying the shear lag model leads to the conclusion that the latter tends to overestimate the constraint effect of the neighbouring fibres when the confinement effect is large $(R e / r<3)$.

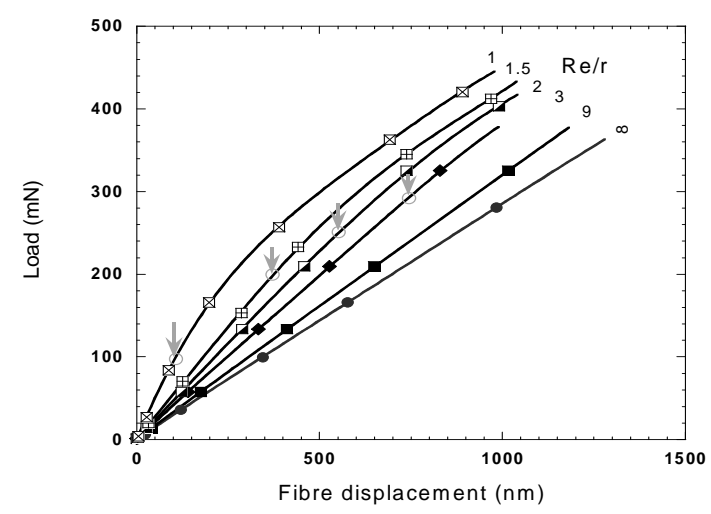

Figure 7. Load-fibre displacements results obtained by FEM, for fibres with different constraint factors Re/r, assuming a perfect interface. As the constraint increases (lower Re/r), the stiffness of the response increases.

This is shown in figure 8 where the parameter $n$ as obtained from the slope of the FEM load-displacement curves and from equation (2) is plotted as a function of the confinement. The shear lag model overestimates the confinement effect of the surrounding fibres on the stiffness of the load-displacement curve as much as $30 \%$, for $\mathrm{Re} / \mathrm{r}<3$.

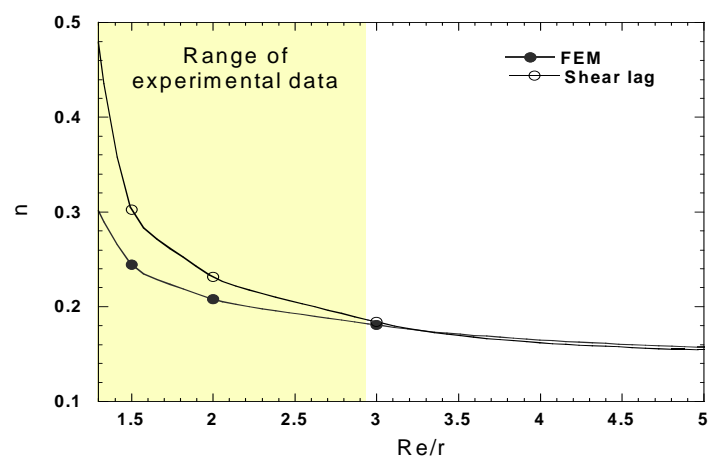

Figure 8. Comparison between the shear lag model results and the FEM results. Value of $n$ versus Re/r as measured by FEM and as calculated using equation (2).

\subsection{Effect of the interfacial strength}

The load-displacement curves obtained by FEM are plotted in figure 9 for fibres with different constraint factors $R e / r$ and an interfacial strength of $80 \mathrm{MPa}$. The dashed line indicates the equivalent behaviour assuming a perfect interface.

In all cases, the departure of linearity, as indicated by the gray arrows, marked the onset of debonding of the indented fibre, even if the interfacial shear strength was comparable to the matrix shear yield stress. An interesting situation would be that in which the matrix shear yield stress were lower than the interfacial strength, which will be the subject of future studies.

Finally, figure 10 shows the comparison between one experimental push-in test and the corresponding FEM simulation for different values of the constrain factor $R e / r$. A perfect match is obtained in this case for $R e / r=1.5$. It is worth mentioning at this point that the same value of interfacial strength, i.e. $80 \mathrm{MPa}$, was obtained using the shear lag model, but with $R e / r=2$. 


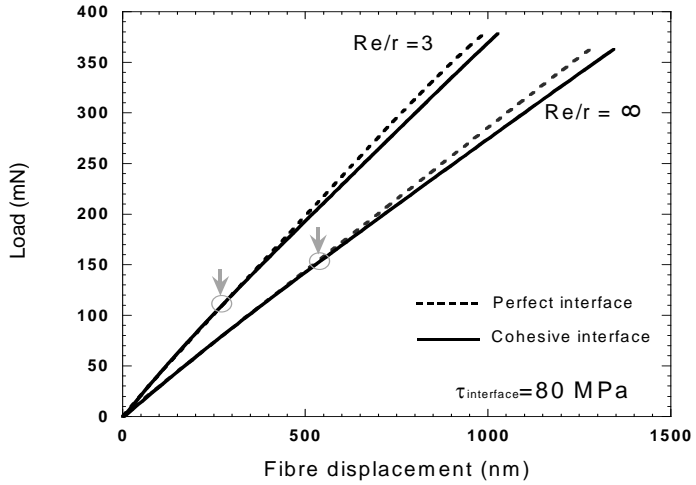

Figure 9. Load-fibre displacements results obtained by FEM, for fibres with different constraint factors Re/r, assuming a perfect interface and a cohesive interface with an interfacial strength of $80 \mathrm{MPa}$.

This means that the simplified shear lag models used constitute an appropriate method to analyse the push-in test, provided that the parameter $(R e / r)$ is obtained by fitting the linear slope of the load displacement curve using equations (1) and (2). However, it should be stressed out that the values of $(\mathrm{Re} / \mathrm{r})$ obtained in this way act as fitting parameters and are larger than the real distance to the neighbouring fibres, because the shear lag model overestimates the constrain effect, as shown in figure 8 .

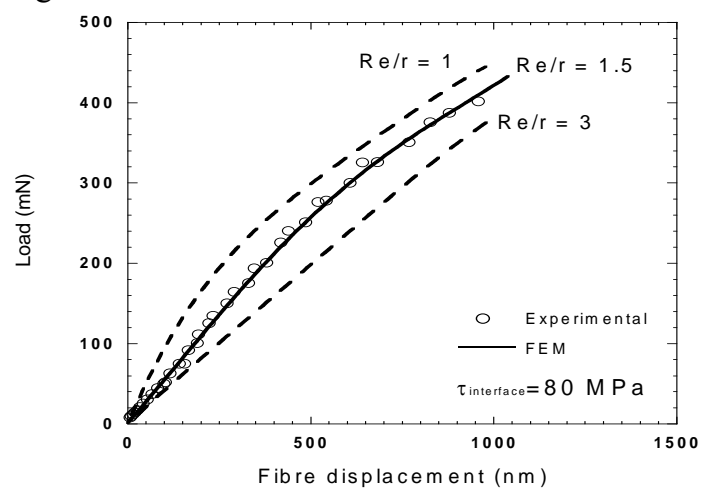

Figure 10. Comparison between experimental results on a 20 $\mu m$ diameter fibre and FEM results with different Re/r and an interfacial strength of $80 \mathrm{MPa}$. A perfect match is obtained for $\operatorname{Re} / r=1.5$.

\section{CONCLUSIONS.}

A detailed micromechanical modelling of the push-in test, coupled with experimental tests, was carried out in a glass-fibre reinforced epoxy matrix composite using nanoindentation. The model took into account the interfacial fracture process using interface cohesive elements at the fibre-matrix interface and focused on the constrain effects of the nearest neighbouring fibres to determine the validity of the simplified shear lag models currently used to interpret the results of the test. The results showed that the analytical models currently used are valid when the indented fibre is relatively isolated from its neighbours. For closely packed fibres $(\mathrm{Re} / \mathrm{r}<3)$, which is the normal situation in polymer matrix composites, the shear lag model tends to overestimate the constraint effect of the neighbouring fibres. However, according to the FEM results, the simplified shear lag models used constitute an appropriate method to analyse the push-in test even in this case, provided that the parameter $(R e / r)$ is obtained by fitting the linear slope of the push-in load displacement curve. In this case, the values of $(\mathrm{Re} / \mathrm{r})$ obtained act as fitting parameters and are larger than the real distance to the neighbouring fibres. The simulations also showed that plasticity effects in the matrix are not negligible for large local volume fraction of fibres and can give rise to erroneous interpretations of the push-in test. The results showed that in the case of the composite tested, with comparable matrix shear yield stress and interfacial strength, the push-in test can be applied safely to determine the interfacial strength. Further work is needed to determine the relative matrix shear yield stress to interfacial strength ratios at which this is not the case.

\section{RERERENCES}

[1] Cox, B., Yang, Q., 2006. Science 314, 1102-1107.

[2] González, C., LLorca, J. 2006. Acta Mater., 54, 4171.

[3] Saud A., Ghydaa A., Safaa A.R. 2009. Materials and Design 30, 1835-1840.

[4] González, C., LLorca, J. 2001. Acta Mater., 49, 3505 .

[5] Rollin, M., Jouannigot, S., Lamon, J., Pailler, R. 2009. Composites Science and Technology 69, 14421446.

[6] Mandell, J.F., Chen, J.H. and McGarry, F.J. 1980. Znt. J. Adhes. Adhes. I, 40.

[7] Kalinka, G., Leistner, A. and Hampe, A. 1997. Composites Science and Technology 51, 845-851

[8] Kharrat, M., Chateauminois A., Carpentier, L. and Kapsa, P. 1997, Composite Part A 28A, 39-46.

[9] Zidi, M., Carpentier L., Chateauminois A., Sidorof, F. 2000. Composites Science and Technology 60, 429437.

[10] Cox, H.L. 1942, Br. J. Appl. Phys. 3, 72.

[11] Kelly, A. and Tyson, W.R. 1965. J. Mech. Phys. Solids 13, 329.

[12] Desaeger, M. and Verpoest. I. 1993. Compos. Sci. Technol. 48, 215.

[13] Molina-Aldareguia, J.M., Rodríguez, M., González, C., LLorca, J. 2009, in preparation.

[14] Gonzalez, C., LLorca, J. 2007b. Composites Science and Technology 67, 2795-2806.

\section{ACKNOWLEDGEMENTS}

This investigation was supported by the European Union through the project MAAXIMUS and by the Comunidad de Madrid and the ERA-NET MATERA through the program DEFCOM. The composite materials used in this investigation were manufactured at INTA (Instituto Nacional de Técnica Aerospacial), and the authors want to express their gratitude to Dr. J. M. Pintado and to Dr. M. A. de la Torre. 